Ann. Biol. anim. Bioch. Biophys., I969, 9 (2), 273-28o.

\title{
ÉTUDE ULTRASTRUCTURALE DU SITE DE CONSERVATION DES SPERMATOZOÏDES DANS L'OVAIRE DE POECILIA RETICULATA (POISSON TÉLÉOSTÉEN)
}

\author{
B. JALABERT, R. BILLARD \\ avec la collaboration technique de Anne-Marie EscAfFre \\ Station centrale de Physiologie animale, \\ Centre national de Recherches zootechniques, 78 - Jouy-en-Josas \\ Institut national de la Recherche agronomique
}

\section{SOMMAIRE}

La lumière ovarienne du Guppy présente, dans la partie antéro-dorsale, un ou plusieurs diverticules alvéolés qui forment un réceptacle séminal assurant une longue survie des spermatozoïdes. Ceux-ci sont profondément enfoncés dans le cytoplasme des cellules épithéliales des alvéoles du réceptacle ; l'aspect ultrastructural de ces " cellules hôtes " ainsi que le mode d'insertion des spermatozoïdes permettent de les comparer aux cellules de Sertoli, dans le testicule. Leur dégénérescence, spontanée ou induite, pourrait être l'un des mécanismes responsables de la libération des spermatozoïdes fécondants.

Il est bien connu que certaines femelles de Téléostéens vivipares de la famille des Poecilidae peuvent donner naissance à plusieurs portées successives à la suite d'une seule insémination, grâce à la conservation dans l'ovaire de spermatozoïdes susceptibles de garder plusieurs mois leur pouvoir fécondant. Ce phénomène a été rapporté en I908 par PhILIPPI chez Glaridichtys. Winge (I937) a observé un maximum de 8 portées successives à la suite d'une seule insémination, ce qui correspondrait à une durée de conservation de 8 à ro mois atu plus. VAN OORDT (I928) a trouvé une durée du même ordre chez Xiphophorus.

Toutefois, peu d'auteurs se sont intéressés à la structure du lieu de stockage des spermatozoïdes, et encore moins aux mécanismes physiologiques éventuellement liés à leur survie et à leur libération. Après insémination, il semble y avoir dissociation rapide des spermatozeugmes (PHILIPPI, I908), les spermatozoïdes n'effectuent alors qu'un bref transit dans l'oviducte et pénétrent dans la lumière ovarienne constituée par des digitations irrégulières en rapport d'une part avec l'oviducte, et d'autre part avec les entonnoirs de fécondation des ovocytes ; c'est dans une crypte de la paroi dorsale de cette lumière que Chambolie (I963), chez Lebistes, observe la conservation des spermatozoìdes, et au moment de la parturition, leur libération 
dans l'ensemble de la lumière ovarienne d'où un certain nombre gagne ensuite les entonnoirs de fécondation. Il n'existait pas à notre connaissance d'étude plus précise de ces sites d'accueil des spermatozoïdes, en ce qui concerne les Poecilidae.

Cependant, l'aptitude à la conservation à long terme des spermatozoides par la femelle après insémination, se rencontre chez d'autres vertébrés, où le problème a parfois été mieux étudié. Ainsi, les femelles des Amphibiens Urodèles possèdent au niveau de la paroi dorsale du cloaque un organe multitubulaire décrit depuis longtemps par de nombreux auteurs (revue par Jol,Y, I960), et servant de réceptacle séminal. JoLy (I960), après BAyLIS (1939) a montré que la femelle de Salamandre est susceptible d'être féconde 2 ans $x / 2$ après un accouplement. Parmi les Reptiles, des durées de conservations extrêmes de l'ordre de 4 à 6 ans ont été rapportées (HAINES, I940 ; Fox, I956), mais la durée la plus fréquemment observée est de quelques mois, de l'automne au printemps suivant chez Tamnophis sirtalis (Fox, I956) ainsi que chez Vipera aspis (SAINT-GIRONS, I957).

Chez les Oiseaux, la durée de survie des spermatozoides est beaucoup plus courte : quelques semaines au maximum; certains sont libérés en petit nombre dans l'oviducte lors de chaque ovulation (BoBR et al., I964 $b$; VAN KREY, I964); une fois libérés, leur survie est alors réduite à quelques heures. Les sites de conservation en cause sont essentiellement les glandes tubulaires situées à la jonction utéro-vaginale (BOBR, I962; BOBR et $a l .$, I964 $a$; LORENZ, I966) et éventuellement d'une manière secondaire, d'autres glandes situées à la jonction du pavillon et de l'infundibulum (VAN Drimmelen, I946 ; FujII et TAMURA, I963 ; Bobr et al., I964 a) ; pour SchinDLER et al. (I967) les deux types de glandes seraient équivalents.

Enfin, parmi les Mammifères, 1a Chauve-Souris femelle conserve les spermatozoïdes après accouplement d'automne, pendant toute la durée de son hibernation, grâce au rôle nourricier de l'épithélium utérin (CourriER, I920-I92I ; HaRTManN, I933).

Dans tous les cas, cette conservation des spermatozoïdes dans les voies génitales femelles pose le problème du déterminisme de leur survie et de leur libération au moment de la fécondation. Avant toute expérimentation dans ce sens, chez Poecilia reticulata, nous avons essayé d'établir avec précision les relations morphoiogiques existant entre les spermatozoïdes et les tissus maternels qui les accueillent comme cela a déjà été réalisé en microscopie électronique chez la Poule (VAN KREY et al., I967; SchINDLER et al., I967) et chez la Chauve-Souris (NAPOLITANo, KRUTzSCH et WimsaTT, I963; WIMSATT, KRUTZSCH et NAPOLITANO, I966).

\section{MATÉRIEL E'T MÉTHODES}

L'ovaire de femelles de Poecilia reticulata en pleine activité sexuelle a été prélevé après anesthésie au MS 222 Sandoz et placé directement dans une solution à $4 \mathrm{p}$. I00 de glutaraldéhyde dans du tampon phosphate à $\mathrm{pH} 7,25$ (SABATINI et al., I962) où il a été disséqué pour isolement de fragments de la paroi dorsale de la cavité ovarienne. Après une heure de préfixation, les pièces séjournent I heure dans une solution d'acide osmique à 2 p. Ioo dans le même tampon (MILLONIG, 1962).

L'inclusion a été effectuée dans l'épon, et les coupes ont été colorées $5 \mathrm{mn}$ à l'acétate d'uranyle et Io mn au citrate de plomb puis recouvertes d'un film de carbone. 


\section{RÉSULTATS}

En microscopie photonique, "le réceptacle séminal " de la femelle de Poecilia reticulata apparaît constitué par une ou plusieurs cryptes en cul-de-sac dans la région antérodorsale de la lumière ovarienne (fig. I), en continuité avec celle-ci. Elles s'en

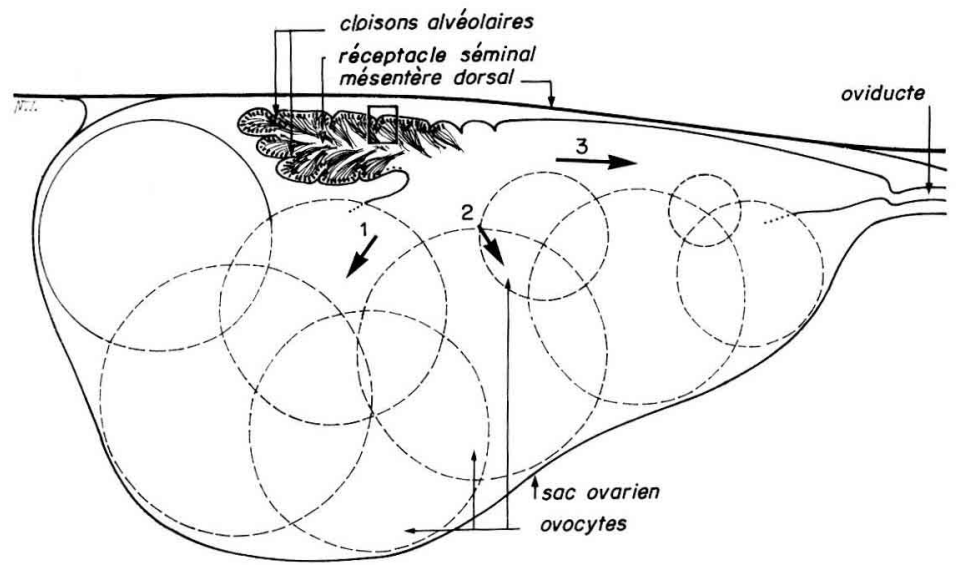

FIG. I. - Représenlalion schématiqus, en coupe sagitlale, d'une partie du réceplacle séminal, chez un animal jeune, avec ovaire immature (ovocytes de $600 \mu$ au maximum). Chez une femelle en pleine reproduction, le réceptacle serait écrasé entre les gros ovocytes et les follicules embryonnés. Des replis épithéliaux formant cloisons délimitent des alvéoles, mais le réceptacle reste largement en continuité avec la cavité ovarienne (flèches I et 2) ainsi qu'avec l'oviducte (flèche 3 ).

Dans le rectangle : partie de l'épithélium du réceptacle au niveau d'une cloison alvéolaire agrandie sur la figure 2. (Voir la liste des abréviations en fin d'article).

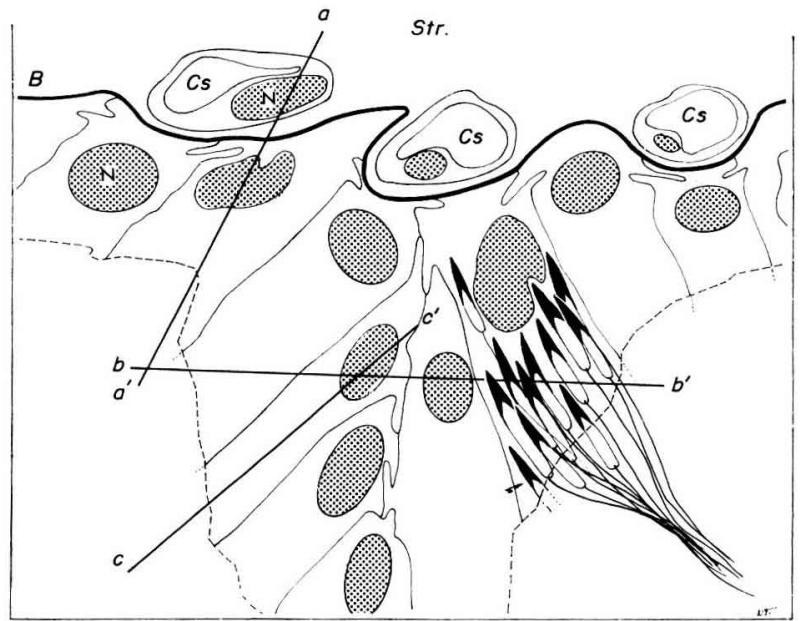

Fig. 2. - Représentation schématique d'une coupe au niveau d'une cloison alvéolaire (rectangle sur la fig. נ), reconstituée a partir d'observations sur des plans de coupe différents en microscopie électronique. Les spermatozoìdes n'ont été représentés que dans une seule cellule pour ne pas alourdir le schéma $\left(a a^{\prime}, b b^{\prime}\right.$, et $c c^{\prime}$ : plans de coupe des photographies suivantes).

(Voir la liste des abréviations en fin d'article). 
distinguent par une série de replis de l'épithélium qui cloisonnent le réceptacle en alvéoles plus ou moins profondes, largement ouvertes sur la lumière. Les spermatozoỉdes sont groupés en faisceaux ou en paquets épais dans ces alvéoles, la tête orientée vers l'épithélium, très proche des noyaux cellulaires sans que l'on puisse déterminer avec certitude la nature de leurs relations morphologiques.

En microscopie électronique (fig. 2, planches I, II, III, IV) on constate que les spermatozoïdes sont effectivement enfoncés dans le cytoplasme des cellules épithéliales, parfois profondément. Ces "cellules réceptrices " forment généralement un épithélium unistratifié mais se chevauchent en formant des replis au niveau des cloisons alvéolaires. Elles sont séparées du stroma conjonctif ovarien environnant par une basale contre laquelle sont en outre appliqués, côté stroma, de nombreux capillaires sanguins (planche I). Cette basale est absolument continue et se double, par endroits seulement, de fibrilles de collagène (planche III, B). Pour une description commode, on peut distinguer 2 parties dans chaque cellule réceptrice : partie basale contenant le noyau, et partie apicale pénétrée par de très nombreux spermatozoïdes, du côté de la lumière du réceptacle.

Au niveau de la partie basale, ces cellules présentent entre elles des interdigitations cytoplasmiques profondes, et montrent, en outre, de très nombreux desmosomes d'où partent par endroits des réseaux denses de tonofibrilles (planche III B). Le noyau, gros et contourné, parfois multilobé (planche II) est très proche de la basale; à son voisinage, les mitochondries sont abondantes ainsi que le long de la basale. Des formations golgiennes très développées sont bien réparties dans l'ensemble du cytoplasme ; le réticulum endosplasmique ne semble pas particulièrement abondant. Quelques têtes de spermatozoïdes arrivent au niveau du noyau, certaines le contournent, atteignant ainsi directement la basale qu'elles ne semblent jamais franchir.

En partie apicale, les spermatozoïdes disposés parallèlement sont extrêmement nombreux ; chacun d'eux est emprisonné dans les mailles d'un réseau cytoplasmique très resserré vers la partie basale (planche III C), s'élargissant progressivement vers la lumière, et se terminant par des prolongements cytoplasmiques irréguliers, contournés ou repliés, entre lesquels sont regroupés des faisceaux de flagelles, des pièces intermédiaires ou même des têtes (planche I).

Enfin, il est à noter que certaines cellules réceptrices montrent parfois des signes de dégénérescence très nets : de nombreuses vésicules intracellulaires apparaissent (planche IV A) envahissant progressivement tout le cytoplasme ; le réseau emprisonnant les spermatozoïdes paraît se relâcher. En partie apicale, le cytoplasme devient fluide et s'écoule dans la lumière du réceptacle en entraînant les spermatozoïdes (planche IV B). Enfin, les cellules se décollent complètement de la basale, le noyau lui-même étant expulsé dans la lumière avec les spermatozoïdes et les restes cytoplasmiques. Cette dégénérescence ne semble affecter, à un moment donné, que certains groupes de cellules, les autres cellules réceptrices ayant 1'aspect normal précédemment décrit. Par ailleurs, nous avons observé un taux de dégénérescence très faible parmi les spermatozoïdes conservés ; mais il nous a paru absolument indépendant de l'état des cellules hôtes. Ces spermatozoïdes conservés chez la femelle ne présentent pas de différences morphologiques par rapport à ceux que nous avons observés chez le mâle. 


\section{DISCUSSION}

L'existence de rapports morphologiques étroits entre les cellules du réceptacle séminal de la femelle et les spermatozoïdes conservés, avec pénétration d'un certain nombre d'entre eux dans le cytoplasme de cellules hôtes, a déjà été mise en évidence chez certaines femelles de vertébrés : c'est ainsi que Joly (I960), chez la Salamandre, a observé que les têtes de spermatozoïdes sont enfoncées dans les cellules épithéliales cylindriques du réceptacle. Ces cellules ont, en outre, un caractère glandulaire et libèrent dans la lumière un produit de sécrétion granuleux. NAPOLITANo et al. (I963) ont constaté l'inclusion des spermatozoïdes dans les cellules de l'épithélium utérin. Cependant, chez le Reptile Thamnophis sirtalis, Fox (I956) observe en microscopie photonique les spermatozoïdes simplement au contact d'un épithélium très bas, (alors qu'il est haut en 1'absence de spermatozoïdes), mais pénétrant quelquefois dans des vacuoles apicales. Enfin, chez la Poule, la tête spermatique apparaît parfois fixée dans le cytoplasme des cellules des glandes infundibulaires (BOBR et al., I964a; FuJII, I963), mais dans les glandes utéro-vaginales où la conservation serait meilleure, il n'y a pas de contacts aussi étroits (FUJII, I963); dans les deux cas, les spermatozoïdes sont cependant enserrés par de nombreuses microvillosités cellulaires (VAN KREY et al., I967) et parfois très proches des cellules (SchINDLER et al., I967).

En fait, suivant les espèces les conditions physiologiques liées à la survie à long terme (nutrition, inhibition de la motilité, etc.) peuvent se trouver réunies aussi bien dans le cytoplasme de cellules hôtes particulières que dans la lumière du réceptacle grâce à une activité sécrétrice des cellules épithéliales. En ce qui concerne Poecilia reticulata, il nous semble que la pénétration dans les cellules décrites n'est pas simplement un phénomène secondaire dû à l'activité propre des spermatozoïdes, car, d'une part cette particularité est étroitement localisée à une partie déterminée de la cavité ovarienne sans réaction de défense apparente du tissu intéressé, et, d'autre part, 1a proportion de spermatozoïdes libres dans l'ovaire est très faible, excepté à certains moments priviligiés du cycle, après parturition, ou après insémination.

I1 existe une certaine analogie ultrastructurale entre ces cellules-hôtes et les cellules de Sertoli chez le mâle (BILLARD, I96g), tant du point de vue de leur ultrastructure que du mode d'insertion respectif des spermatozoildes et des spermatides allongées. Jol, (Ig6o) chez la Salamandre, et NAPolitano et al. (I963) chez la Chauve-Souris avaient déjà effectué une telle comparaison entre les cellules hôtes de la femelle et les cellules de Sertoli du testicule. Chez Poecilia, cette analogie peut d'ailleurs aller jusqu'au mécanisme de libération des spermatozoïdes par dégénérescence des cellules de Sertoli et leur écoulement,dans la lumière du canal efférent. Chez la femelle, le phénomène pourrait en outre, être favorisé par une action mécanique à parturition, ou par la contraction de fibres musculaires lisses abondantes dans l'ovaire. L'hypothèse d'un tel mécanisme de libération est séduisante. Si elle correspond à la réalité, le problème de son déterminisme reste posé : s'agit-il d'un phénomène inéluctable lié au vieillissement des cellules hôtes aboutissant à la libération continue d'un certain nombre de spermatozoïdes, ou bien est-il lié en importance à la maturation ovocytaire et à la période de fécondation et provoqué par un stimulus endocrinien donné ? 
Enfin, il n'est pas certain que les spermatozoïdes soient absolument passifs dans leur libération et l'hypothèse de leur activation directe par un stimulus biochimique au moment de la fécondation (BAILEY, I933 ; CHAMBOLLE, I963) n'est pas à exclure.

Rę̧u pour publicalion en décembre 1968.

\author{
SUMMARY \\ FINE STRUCTURE OF THE STORAGE SITES OF SPERMATOZOA \\ IN THE GUPPY " POECILIA RETICULATA "
}

The ovarian lumen of the Guppy antero-dorsally comprises one or several alveolate diverticuli forming a seminal receptacle, within which spermatozoa are inserted in the epithelial cell cytoplasm.

The basal part of these cells, containing large, sometimes multilobular nucki, is lined by a continuous membrane separating them from the surrounding connective stroma in which numerous blood capillaries course (picture 1 ). The basal cytoplasms of adjacent cells interdigitate to a great extent (picture 2) and cell membranes are bound with numerous desmosomes (pictures $2,3 \mathrm{~b}$ ).

The apical cytoplasm, packed with spermatozoa, occurs in reticular form and loosens towards the lumen.

The degeneration process of epithelial cells, i. e. cytoplasmic vacuolizing (pictures $3 c, 4 a$ ) and outflowing $(3 b)$ into the lumen, seems to partake of the mechanism responsible for the releasing of fertilizing spermatozoa.

\title{
RÉFÉRENCES BIBLIOGRAPHIQUES
}

BaILey R. J., 1933. The ovarian cycle in the viviparous teleost Xiphophorus helleri. Biol. Bull., 64, 205-224.

Baylis H. A., 1939. Delayed reproduction in the spotted Salamander. Proc. Zool. Soc. London, A, 109, 243-246.

Billard R., 1969. La spermatogenèse de Poecilia reticulata. IV. Ultrastructure des cellules de Sertoli. Ann. Biol. anim. Bioch. Biophys. (主 paraître)

BOBR L. W., LoRenz F. W., OGaSAWARA F. W., 1962. The role of the uterovaginal junction in storage of cock spermatozoa (Abstract) Poult. Sci., 41, , 628.

Bobr LL. W., Lorenz F. W., Ogasawara F. X., Ig64a. Distribution of spermatozoa in the oviduct and fertility in domestic birds. I. Residence sites of spermatozoa in fowl oviducts. J. Reprod. Fert., 8, 39-47.

Bobr L. W., Ogasawara F. X., LoRenz F. W., $1964 b$. Distribution of spermatozoa in the oviduct and fertility in domestic birds. II. Transport of spermatozoa in fowl oviduct. J. Reprod. Fert., 8, 49-58.

Crambolle P., 1963. Rerherches descriptives et expérimentales sur l'appareil reproducteur et la physiologie de la reproduction de Lesbites reticulatus (PETERS). Thèse doct. $3^{\mathrm{\theta}}$ cycle Bordeaux, $94 \mathrm{p}$

CourRIER R., 1920. Sur l'existence d'une sécrétion de l'épithélium utérin chez la Chauve-Souris hibernante, sa signification. C. R. Soc. Soc. Biol., 83, 243-244.

Courrier R., Ig2I. Sur le rôle physiologique des sécrétions utérines et tubulaires chez la Chauve-Souris hibernante. C. R. Soc. Biol. 84, 572-574.

Fox W., 1956. Seminal receptacles of snakes. Anat. Rec., 124, 519-539.

FujII S., TAMURA T., I963. Location of sperms in the oviduct of the domestic fowl with special reference to storage of sperms in the vaginal glands. F. Fac. Fish. Anim. Husb. Hiroshima Univ., 5, I 45-163.

HAINES T. P., I940. Delayed fertilization in Leptodeira annulata polystica. Copeia, 1940, I $16-118$.

Hartmann C. G., 1933. On the survival of spermatozoa in the female genital tract of the Bat. Quart. Rev. Biol., 8, 185-193.

JoLY J., rg6o. La conservation des spermatozoïdes et les particularités histophysiologiques du réceptacle séminal chez la Salamandre Salamandra salamandra taenatia. C. R. Acad. Sci. Paris, 250, 2269-2271. 
LORENz F. W., 1966. Behavior of spermatozoa in the oviduct in relation to fertility. In Phvsiology of the domeslic towl (chap. 6). Edited by C. Horton-Smith and E. C. Amoroso Publ. Oliver and Boyd Ltd Edinburgh 1966.

Millonig G., I962. Further observations on a phosphate buffer for osmium solutions. in S. J., BRESSE. Electron microscopy, 5th Intern. Cong. Electr. Micr., 2, 8. Acad. Press New York and London.

Napolitano L. M., KRUtzsch P. H., WimsatT W. A., 1963. Electron microscopy of the sperm-uterine relationship in a hibernating bat. (Ábstr.) Anat. Rec., 145, 365 .

Philipr E., I908. Fortplanzungsgeschichte der viviparen teleoster Glaridichthys januarius und $G$. decemmaculatus in ihrem Einfluss auf Lebensweise, makroskopische und mikroskopische Anatomie. Zool. Jahr., 27, I-94.

Sabatini D. D., Bensch K. G., Barnetr R. J., I962. New fixatives for cytological and cytochemical studies. in S. J. Bresse, Electron Microscopy, 5th Intern. Congr. Electr. Micr., 2, L3. Acad. Press New York and London.

SaInT-Girons H., I957. Le cycle sexuel chez Vipera aspis dans l'ouest de la France. Bull. Biol. France el Belgique, 91, 284-350.

SCHINDLER H. et $a l$, ${ }^{1967}$. The relation of spermatozoa to the glandular tissue in the storage sites of the hen oviduct. Poult. Sci., 46, 1462-I477.

Van Drimmelen G. C., 1946. "Sperm nests" in the oviduct of the domestic hen. J. S. Afr.vet.med. Ass. 17, 42 .

VAN KREY H. P., I 964. Storage and transport of spermatozoa within the oviduct of the domestic fowl. $\mathrm{Ph} . \mathrm{D}$ Thesis, Univ. Calif.

VAN OORDT G. J., I928. The duration of life of the spermatozoa in the fertilized female of Xiphophorus helleri REgan. Tijdschrift Nederl. Dierk. Vereen., Ser. 3, 77*80.

Wimsatt W. A., Krutzsch P. H., Napolitano L., r966. Studies on sperm survival mechanisms in the female reproductive tract of hibernating Bats. I. Cytology and ultrastructure of intra uterine spermatozoa in Myotis lucifugus. Amer. J. anat., 110, 25-60.

Winge Ö., 1922. A peculiar mode of inheritance and its cytological explanation. J. Genetics, 12, I37-144. WINGE Ö., 1937. Succession of broods in Lebistes. Nature, 140, 467. 


\section{ABRÉVIATIONS UTILISÉES \\ SUR LES FIGURES ET PHOTOGRAPHIES}

\begin{tabular}{|c|c|c|c|}
\hline Basale & B & Manchon mitochondrial .... & $\mathrm{m} . \mathrm{m}$. \\
\hline Capillaire sanguin .... & Cs & Membrane cellulaire...$\ldots$. & $\mathbf{M}$ \\
\hline des cellules- & & Mitochondrie...$\ldots \ldots$ & 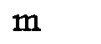 \\
\hline hôtes....... . & $\mathrm{Cy}$ & Noyau & $\mathrm{N}$ \\
\hline Prolongements cytoplas- & & Nucléole ........... & $\mathrm{n}$ \\
\hline miques des cellules-hôtes. & $\mathrm{pCy}$ & Pièce intermédiaire & $\mathrm{Pi}$ \\
\hline Desmosome ............. & $\mathrm{d}$ & Ribosomes ........ & ri \\
\hline Fibres de collagène...$\ldots \ldots$ & fc & Stroma conjonctif ovarien .. & Str \\
\hline Flagelle,$\ldots \ldots \ldots \ldots \ldots$ & $\mathrm{F}$ & Tête de spermatozoïde... & $\mathbf{s}$ \\
\hline Golgi $\ldots \ldots \ldots \ldots \ldots \ldots$ & $\mathrm{g}$ & Tonofibrilles ........ & tf \\
\hline Lumière $d \mathfrak{u}$ réceptacle $\ldots .$. & I & Vésicule $\ldots \ldots \ldots \ldots \ldots$. & $\mathbf{v}$ \\
\hline
\end{tabular}




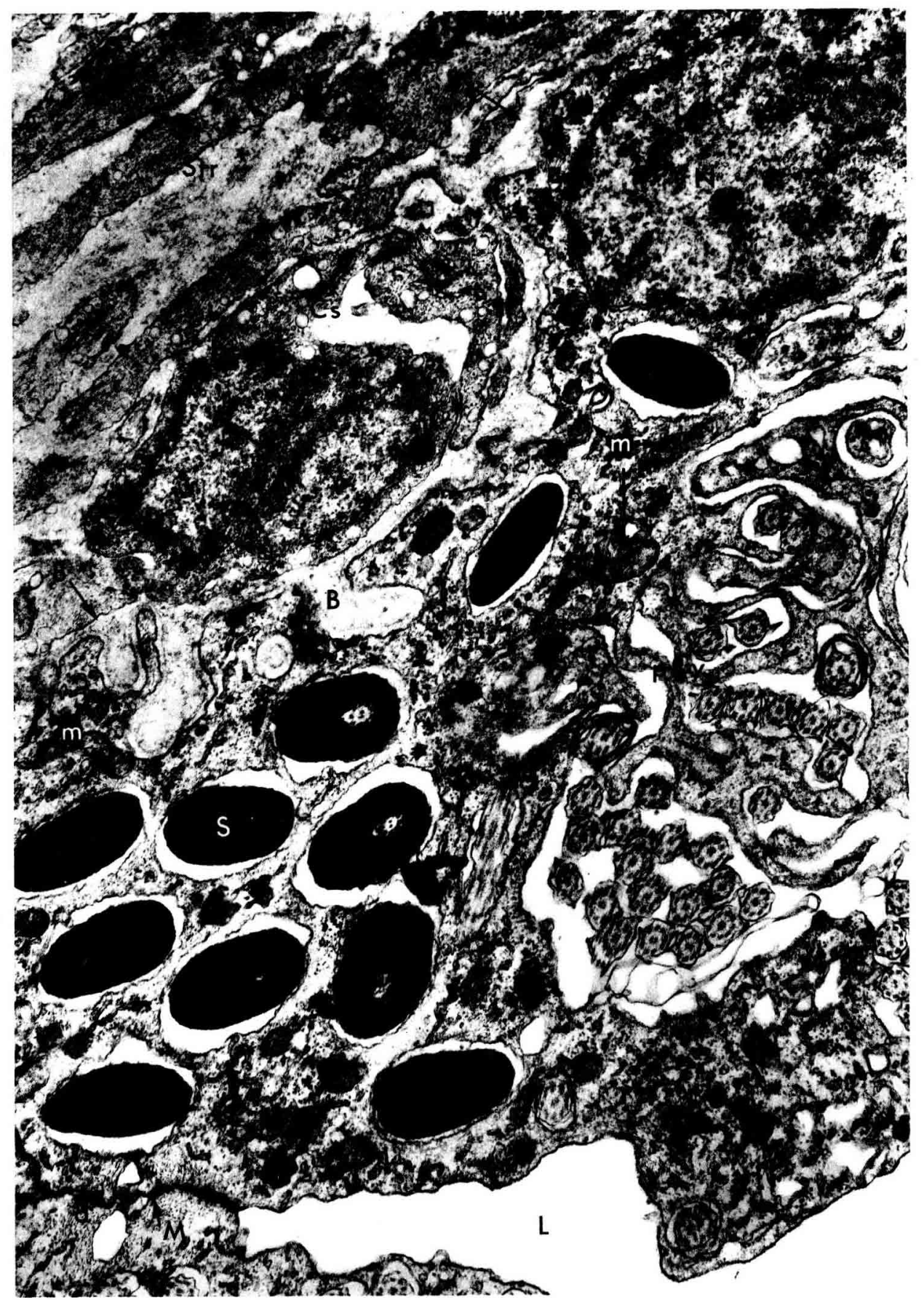




\section{PLANCHE II}

Coupe tangentielle à l'épithélium du réceptacle (bb' sur la fig. 2) montrant des spermatozoldes à tous les niveaux des cellules hotes. Celles-ci sont extrêmement imbriquées. L'un des noyaux (au centre) présente des lobes apparents sur la photographie, séparés par une travée de cytoplasme $(\mathrm{Cy}) .(\times 12.000)$. 
Ann. Biol. anim. Bioch. Biophys., 1969, 9 (2).

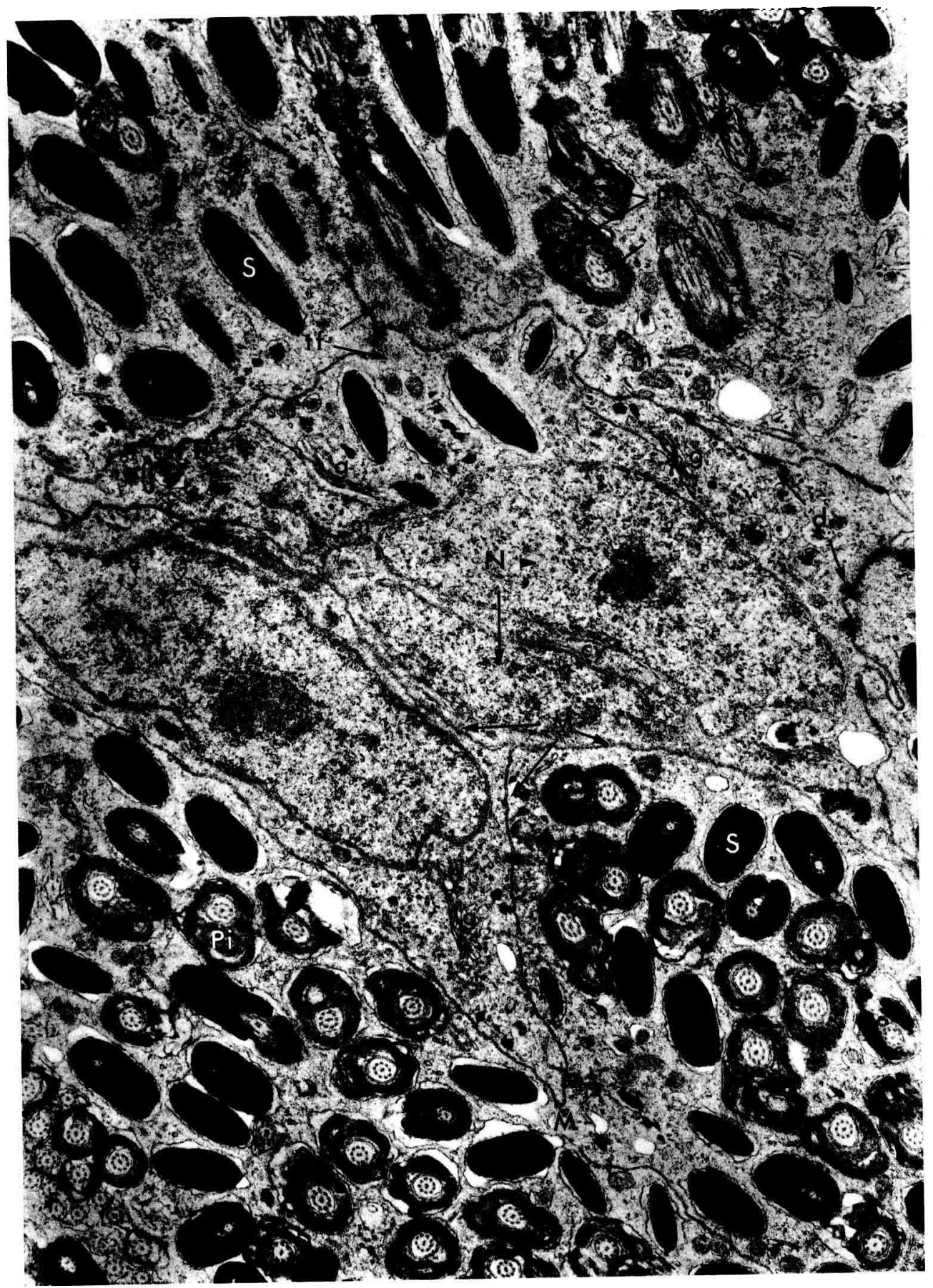

JALABERT et BILLARD. 


\section{PLANCHE III}

A. - Coupe horizontale (cc' sur la fig. 2) d'une cellule hôte (partie basale) avec des têtes de spermatozoldes (S) en coupe sagittale. $(\times 24.000)$.

B. - Coupe subtangentielle par rapport à la basale montrant la jonction entre des cellules hôtes, des desmosomes (d) et un réseau de tonifibrilles (t.f.). La basale est accompagnée ici des fibres de collagène (f.c.). $(\times \mathbf{2 6 . 0 0 0})$.

C. - Réseau cytoplasmique au niveau de pièces intermédiaires. Chacune d'entre elles est entourée par une couche de cytoplasme de la cellule hôte. Celui-ci présente d'ailleurs dans ce cas particulier des signes de vésiculation (V). $(\times 45.000)$. 


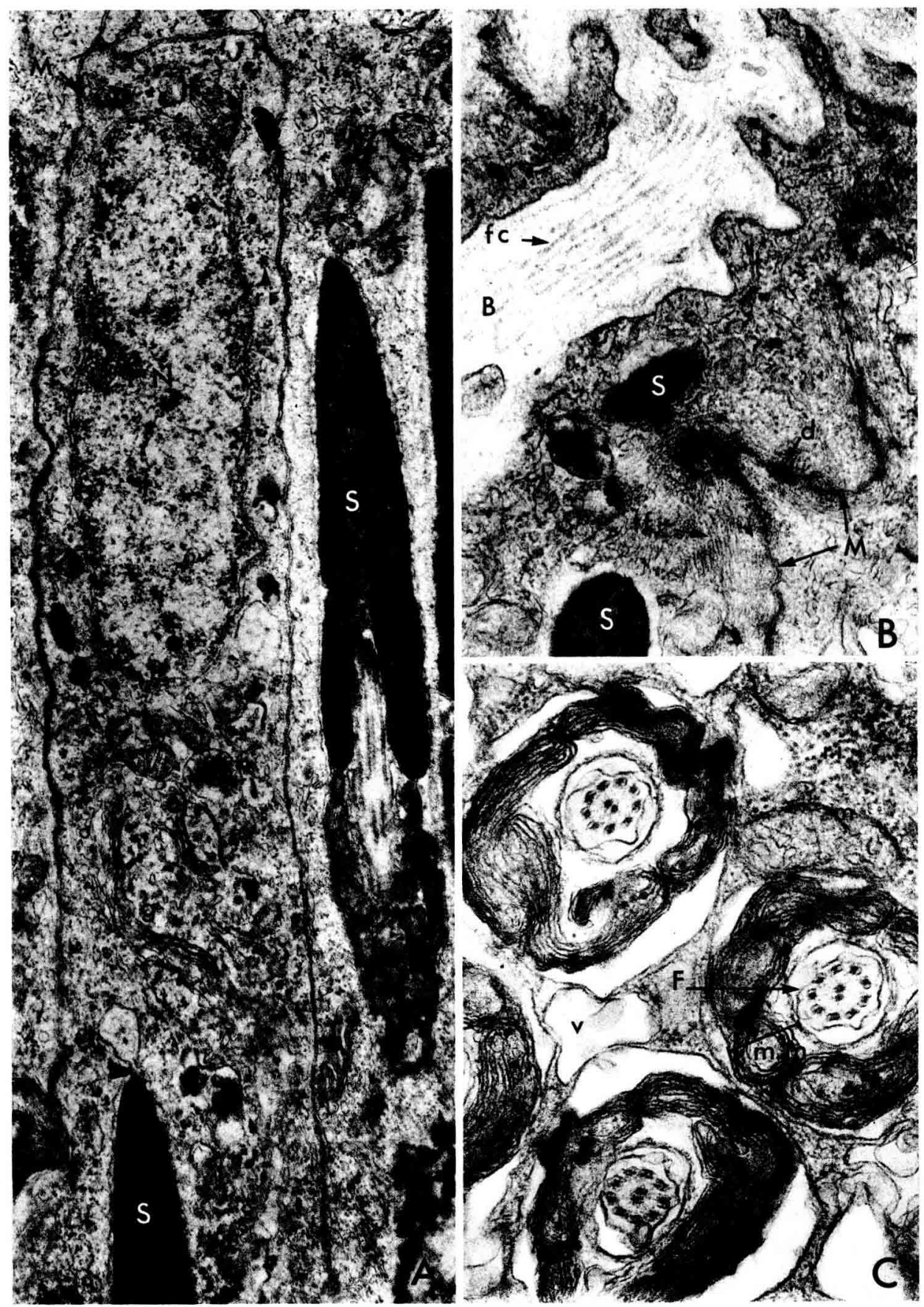




\section{PLANCHE IV}
A. - Cellule hôte en voie de dégénérescence : de nombreuses vésicules (V) apparaissent dans le cytoplasme. Le réseau cytoplasmique entourant les spermatozoldes se relache. Un spermatozolde (Sd) présente un aspect anormal, mais les autres sont apparemment normaux. $(\times 24.000)$.

B. - Ecoulement cytoplasmique dans la lumière du réceptacle avec entrainement des spermatozordes. ( $\times$ 24.000). 


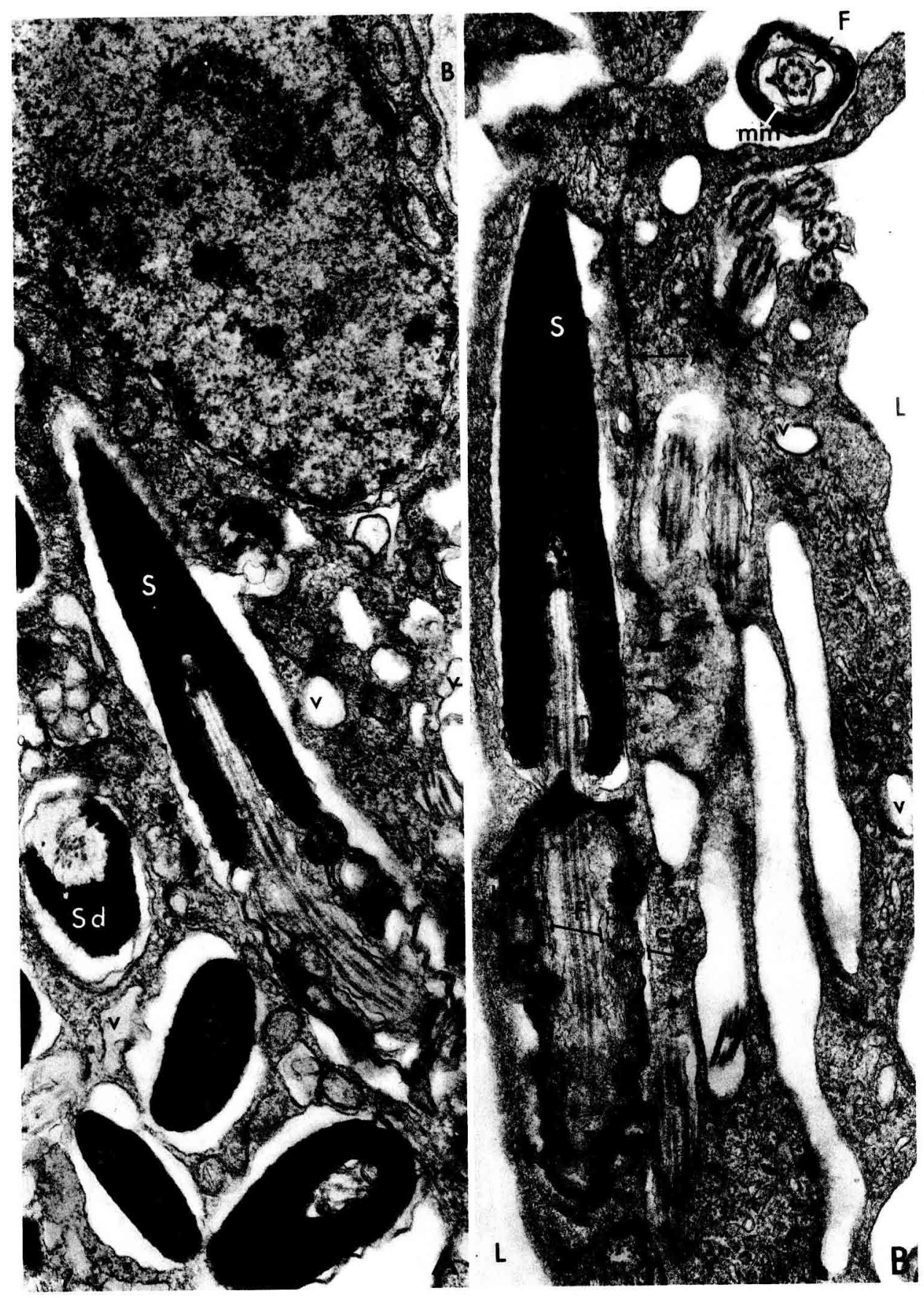

JALABERT et BILLARD. 